\title{
Optimization of back-propagation neural networks architecture and parameters with a hybrid PSO/SA approach
}

\author{
Mahdi Zarei' ${ }^{l}$ Zari Dzalilov ${ }^{2}$ \\ ${ }^{1}$ Hematology Oncology Research Center, \\ Tabriz University of Medical Sciences, \\ Tabriz, Iran. \\ ${ }^{2}$ Centre for Informatics and Applied \\ Optimization, School of Information \\ Technology and Mathematical Sciences, \\ University of Ballarat, Victoria, Australia.
}

mehd i. nategna L 1. com,

Abstract

Determining the architecture and parameters of neural networks is an important scientific challenge. This paper reports a new hybrid optimization method for optimization of back- propagation neural networks architecture and parameters with a high accuracy. We use particle swarm optimization that has proven to be very effective and fast and has shown to increase the efficiency of simulated annealing when applied to a diverse set of optimization problems. To evaluate the proposed method, we employ the PIMA dataset from the University of California machine learning database. Compared with previous work, we show superior classification accuracy rates of the developed approach.

\section{Introduction}

The architecture and parameters of neural networks may usually be determined by trial and error methods, and in some problems a mathematical relation for these has been found. In this paper we present an optimization algorithm for determining the architecture and parameters of back propagation neural networks. Neural networks are a class of biologically inspired adaptive systems consisting of a number of simple processing elements interconnected with each other [1]. Neural networks have been used in functional approximation, pattern recognition, image processing, signal processing, expert systems and other fields [5]. In this paper we consider a multilayer feed-forward neural network that has been widely used in pattern classification. The network consists of an input layer, one hidden layer and an output layer. We train the network using a backpropagation algorithm, which is essentially a gradient descent minimization method. The training is supervised, with learning parameters controlling the training process and minimizing errors between actual and predicted output functions. Parameters and architecture of neural network vary for different applications. The proposed method uses particle swarm optimization algorithm (PSO) and simulated annealing algorithm (SA). The PSO algorithm is used for weight updates in neural networks, while the SA algorithm is used to determine the best architecture and parameters of back-propagation neural networks. In this paper we combine these methods and show that our algorithm may determine both discrete and continuous parameters in neural networks with high accuracy. The architecture and parameters of neural networks may usually be determined by trial and error methods, and in some problems a mathematical relation for these has been found. In this paper we present an optimization algorithm for determining the architecture and parameters of back propagation neural networks [2].Neural networks are a class of biologically inspired adaptive systems consisting of a number of simple processing elements interconnected with each other. Neural networks have been used in functional approximation, pattern recognition, image processing, signal processing, expert systems and other fields [5]. In this paper we consider a multilayer feed-forward neural network that has been widely used in pattern classification. The network consists of an input layer, one hidden layer and an output layer. We train the network using a backpropagation algorithm, which is essentially a gradient descent minimization method. The training is supervised, with learning parameters controlling the training process and minimizing errors between actual and predicted output functions. Parameters and architecture of neural network vary for different applications. The proposed method uses particle swarm optimization algorithm (PSO) and simulated annealing algorithm (SA). The PSO algorithm is used for weight updates in neural networks, while the SA algorithm is used to determine the best architecture and parameters of backpropagation neural networks. In this paper we combine these methods and show that our algorithm may determine both discrete and continuous parameters in neural networks with high accuracy.

\section{Parameters and architecture of Back- propagation neural network}

A back-propagation neural network with one hidden layer is determined by its learning rate, the number of hidden neurons, the momentum term and other features. A simple training algorithm of back-propagation neural network is illustrated in Figure 1. The speed of learning is governed by the leaming rate [7]. If the rate is low, convergence is slow and if the rate is too high, the error oscillates without reaching the minimum. Here, the learning rate is a parameter in the solution vector, 
and is optimized to reach the minimum"error for the network. The weight update formula is:

$$
\Delta w_{i j}(t+1)=\alpha \delta_{i}(t) x_{j}(t)
$$

Where the learning rate $\alpha$ is a real number. In backpropagation neural network with momentum, the weight change is in the direction that is a combination of the current gradient and the previous gradient. The weight update formulas for this network with momentum are:

$$
\Delta w_{i j}(t+1)=\gamma \Delta w_{i j}(t)+\alpha \delta_{i}(t) x_{j}(t)
$$

Where the momentum parameter $\gamma$ is constrained to be in the range from 0 to 1 .

$$
\text { Input layer Hidden layer Output layer }
$$

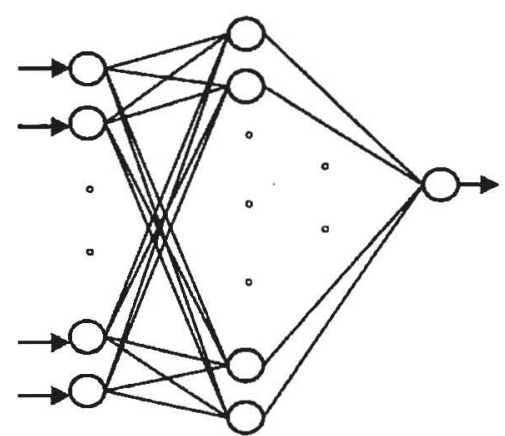

Figure 1: Neural Network with one hidden layer and one output

Here we present a simple training back propagation algorithm for neural networks:

$\mathbf{n}=1$;

Initialize w (n) randomly;

While (stopping criterion not satisfied and $\mathbf{n}<$ max_iterations)

For each example (x, d)

- run the network with input $x$ and compute the output $y$

- update the weights in backward order starting from those of the output layer:

With computed using the (generalized) Delta rule End-for

$$
\mathbf{n}=\mathbf{n}+1
$$

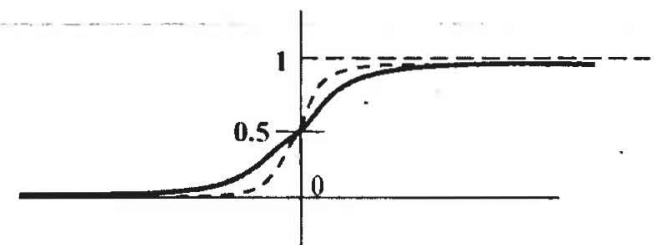

Figure 2: Binary sigmoid function with $\sigma=1$ and $\sigma=.3$

The momentum tends to smooth out small weight error fluctuations, accelerating the descent in steady downhill directions and having a stabilizing effect in directions that oscillate in time [3]. The activation function is another continuous parameter in the network. The activation function for back-propagation neural network should be continuous, differentiable and monotonically nondecreasing. A common activation functions is the binary sigmoid function, illustrated in Figure 2. The steepness of the sigmoid function is modified by a slope parameter $\sigma$ ranging from 0 to 1 [3].

$$
f(x)=1 /(1+\exp (-\sigma x))
$$

With

$$
f^{\prime}(x)=f(x)(1-f(x))
$$

In the topology of neural networks we also need to know the number of hidden neurons. The proposed algorithm can also compute this discrete parameter.

\section{PSO Algorithm}

In a PSO algorithm, the potential solutions, called particles, fly through the problem space by following the current optimum particles [4]. Each particle keeps track of its coordinates in the problem space. The coordinates are associated with the best solution it has achieved so far. This location is called the pbest. Another "best" value that is tracked by the particle swarm optimizer is the best value obtained so far by any neighbor particle. This location is called the lbest. In the special case when a particle takes all the population as its topological neighbors, lbest is called gbest. At each time step of the PSO algorithm, the velocity and position of each particle are updated toward its pbest and lbest locations (local version of PSO). Each acceleration term is weighted by a random number. After finding the two best locations, the particle updates its velocity and position. Figure 3 illustrates the search for a new position in the algorithm. 


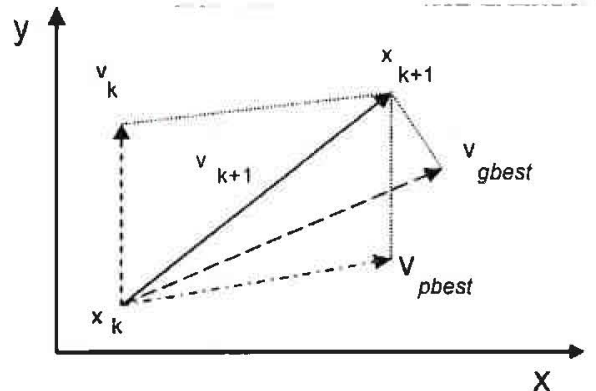

Figure 3: Concept of modification of a searching point by PSO

searching point by PSO. Each particle tries to modify its position using the current positions, the current velocities, the distance between the current position and pbest, and the distance between the current position and the gbest [4]. Here, the velocity and position modifications are illustrated.

$$
\begin{aligned}
& \overrightarrow{v_{i}}(t)=\overrightarrow{v_{i}}(t-1) \\
& +c_{1} \varphi_{1}\left(\text { pbest }-x_{i}(t-1)\right) \\
& +c_{2} \varphi_{2}\left(\text { gbest }-x_{i}(t-1)\right) \\
& \vec{x}_{i}(t)=\vec{x}_{i}(t-1)+\vec{v}_{i}(t)
\end{aligned}
$$

The PSO algorithm has proven both very effective and fast when applied to a diverse set of optimization problems. In the algorithm each particle is randomly positioned in the solution space and is a candidate solution to the optimization problem. Each particle remembers the best position it visited so far, referred to as pbest and the best position by the entire swarm, referred to as gbest [4].

\section{Simulated Annealing Algorithm}

This algorithm uses a temperature schedule. The temperature starts high and then decreases. The algorithm generally assumes that annealing will continue until temperature is zero [8].

The simulated annealing algorithm is shown here:

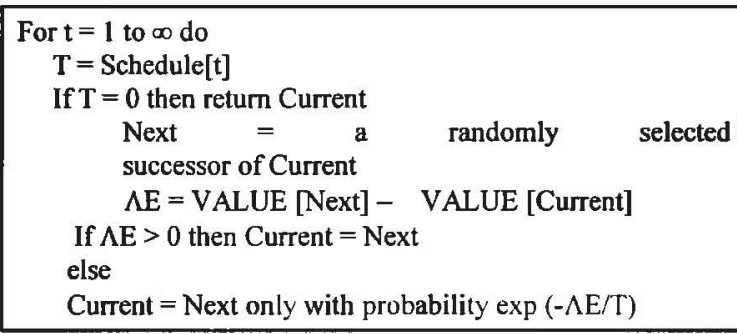

\section{The proposed algorithm for optimization of neural network parameters and architecture}

Given that network parameters may be continuous or discrete, to optimize these parameters we combine the particle swarm optimization and the simulated annealing algorithms. In the first phase, we compute continuous parameters for the given problem, while in the second phase we compute the discrete parameters for that problem.

Suppose we have a solution vector of size $M$. We first initialize the particle population by randomly assigning values for location and velocity. The objective function for each particle is computed in the next step. We then calculate the local best position of each particle, as in a simple PSO algorithm. At this time the $\mathrm{N}$ continuous parameters of the problem are determined. In the next phase these values are held fixed and the remainder of the values in the solution vector are computed.

For computing discrete parameters, we first use the global best of the previous step as the current solution definition. Essentially here we use the simulated annealing algorithm for $\mathrm{M}-\mathrm{N}+1$ discrete parameters. By running the $\mathrm{SA}$ algorithm, we get an epoch and a number of hidden neurons which are used in back propagation network. Continuous parameters may include the learning rate, the momentum term and the slope parameter.

\section{Simulations}

This section evaluates the proposed algorithm using a network with a known architecture and parameters. We use the PIMA dataset with two-category classification from UCI machine learning. There are eight features and one target feature in this dataset. In Table 1 the results of nunning our algorithm for PIMA data set are illustrated:

Table 1: Result of running algorithm for some continuous and discrete parameters

\begin{tabular}{|l|l|l|l|l|}
\hline $\begin{array}{l}\text { Learning } \\
\text { rate }\end{array}$ & $\begin{array}{l}\text { Momentum } \\
\text { term }\end{array}$ & $\begin{array}{l}\text { Hidden } \\
\text { neurons }\end{array}$ & $\begin{array}{l}\text { Sigmoid } \\
\text { factor }\end{array}$ & $\begin{array}{l}\text { Accuracy } \\
\text { rate }\end{array}$ \\
\hline .488 & .297 & 3 & .618 & 82.673 \\
\hline
\end{tabular}

The proposed approach has a classification accuracy rate of 82.7, which is higher when compared with other methods (Table 2). For instance SA+BPN has an accuracy rate of $\mathbf{8 1 . 0}$ [7], and MONNA has an accuracy rate of 76.6 [6].

Table 2: Comparison of the results obtained with those of MONNA

\begin{tabular}{|l|l|l|l|}
\hline Dataset & Proposed Algorithm & SA+BPN & MONNA \\
\hline PIMA & 82.673 & 81.0 & 76.6 \\
\hline
\end{tabular}

The proposed hybrid PSO/SA Optimization Algorithm is summarized as: 
Step I: Initialize the particle population by

$$
\begin{aligned}
& \text { stochastically assigning location } p=\left(\overrightarrow{p_{0}}, \ldots, \vec{p}_{l}, \ldots, \vec{p}_{N}\right) \text { and } \\
& \text { velocity } \quad v=\left(\overrightarrow{v_{0}}, \ldots, \vec{v}_{i}, \ldots, \vec{v}_{N}\right)
\end{aligned}
$$

Step2: Evaluate the fitness of particles:

$$
F(p)=\left(F\left(\overrightarrow{p_{0}}\right), \ldots, F\left(\overrightarrow{p_{i}}\right), \ldots, F\left(\overrightarrow{p_{N}}\right)\right)
$$

Step 3: Keep track of the locations where each individual had its highest fitness so far:

$$
p=\left(\overline{p_{0}{ }^{\text {best }}}, \ldots, \overline{p_{i}^{\text {best }}}, \ldots, \vec{p}_{N}{ }^{\text {best }}\right)
$$

Step 4: Keep track of the position with the global best fitness: $\vec{p}_{g}^{\text {hest }}=\max \vec{p} \in(F(\vec{P}))$

Step 5: Modify the particle velocity based on the previous best and global best positions:

$$
\vec{v}_{i}^{\text {new }}=v_{i}+\varphi_{1}\left(\vec{p}_{i}^{\text {best }}-\vec{p}_{i}\right)+\varphi_{2}\left(\vec{p}_{g}^{\text {best }}-\vec{p}_{i}\right)
$$$$
\text { For } 1 \leq i \leq N
$$

Step 6: Update the particle location:

$$
\vec{p}_{i}^{\text {best }}=\vec{p}_{i}+\vec{v}_{i} \text { best } \text { for } 1 \leq i \leq N
$$

Step 7: If global minimum found go to step 8 Else go to step 2

Step 8: $p_{\text {current }}=\left(\overrightarrow{p^{g b s t}}, \overrightarrow{p_{i}^{g b e s t}}, \ldots,{\overrightarrow{p_{N}}}^{\text {gbest }}, \vec{p}_{N+1}, \ldots, \vec{p}_{M}\right)$

And compute fitness function of $p_{\text {current }}$ :

$$
F\left(\overrightarrow{p_{\text {current }}}\right)
$$

Step 9: while Temprature $\geq$ Temprature_min do step 10 to step 11

Step 10: Compute next of $p_{\text {nexl }}$ solution from $p_{\text {current }}$ and its fitness function: $F\left(\overrightarrow{p_{\text {next }}}\right)$

Step 11: If $F\left(\overrightarrow{p_{\text {current }}}\right) \leq F\left(\overrightarrow{p_{\text {next }}}\right)$ then accept $p_{\text {current }}$ Else if

$$
\left\lfloor\operatorname{Rand}()<\exp \left(-\left(F\left(\overrightarrow{p_{\text {current }}}\right)-F\left(\overrightarrow{p_{\text {mext }}}\right)\right) / \text { Temprature }\right)\right\rfloor
$$

Then accept $p_{\text {current }}$ as current solution

Step 12: Terminate if some condition met Step 13: Go to step 2.

\section{Conclusion and Future Work}

In this paper we present a new method for finding the architecture and parameters of a neural network. Our method is based on a hybrid particle swarm optimization algorithm and simulated annealing algorithm. The method is suitable for determining both discrete and continuous parameters, and performs more accurately on the PIMA dataset compared with other algorithms. Other types of particle swarm optimization algorithms and simulated annealing algorithms may be used in proposed algorithm for improving results. This method may also be applied for detection of other types of neural networks.

\section{References}

[1] D. Mitra, F. Romeo and A. Sangiovanni-Vincentelli, "Convergence and finite-time behavior of simulated annealing," Applied Probability Trust, vol. 18, pp. 747$771,1986$.

[2] D.E. Rumelhart, G.E. Hinton, R.J. Williams, "Learning internal representations by error backpropagation", in: D.E. Rumelhart, J.L. McClelland (Eds.), Parallel Distributed Processing, MIT Press, 1986.

[3] Laurence Fausett, "Fundamentals of Neural Networks", Prentice Hall International, Inc. pp. 283-312

[4] J. Kennedy and R. Eberhart, "Particle swarm optimization," in Proceedings of the IEEE International Conference on Neural Networks, Perth, Australia, December 1995, pp. 1942-1945.

[5] K.J. Hunt, D. Sbarbaro, R. Zbikowski, P.J. Gawthrop, "Neural networks for control systems a survey," Automatica 28 (1992) 1083-1112.

[6] O. Lezoray and H. Cardot, "A neural network architecture for data classification," International Journal of Neural Systems, vol. 11, 2001, pp. 33-42.

[7] S.-C. Chen, S.-W. Lin, T.-Y. Tseng and H.-C. Lin, "Optimization of Back-Propagation Network Using Simulated Annealing Approach", 2006 IEEE International Conference on Systems, Man, and Cybernetics October 8-11, 2006, Taipei, Taiwan, pp. 2819-2823

[8] R. L. Yang, "Convergence of the simulated annealing algorithm for continuous global optimization," Journal of Optimization Theory and Applications, vol. 104, no. 3, pp. 691-716, Mar. 2000. 